\title{
Radiation-induced lower cranial nerve palsy in patients with head and neck carcinoma
}

\author{
STEFAN JANSSEN ${ }^{1,2^{*}}$, CHRISTOPH GLANZMANN ${ }^{1}$, BITA YOUSEFI $^{1 *}$, \\ KARL LOEWENICH $^{1}$, GERHARD HUBER $^{3}$, STEPHAN SCHMID ${ }^{4}$ and GABRIELA STUDER ${ }^{1}$ \\ ${ }^{1}$ Department of Radiation Oncology, University Hospital of Zurich, 8091 Zurich, Switzerland; \\ ${ }^{2}$ Department of Radiation Oncology, University of Lübeck, 23562 Lübeck, Germany; ${ }^{3}$ Department of Otorhinolaryngology, \\ Head and Neck Surgery, University Hospital of Zurich, 8091 Zurich; ${ }^{4}$ Department of Otorhinolaryngology, \\ Bethanien Hospital, 8044 Zurich, Switzerland
}

Received January 19, 2015; Accepted March 12, 2015

DOI: $10.3892 / \mathrm{mco} .2015 .558$

\begin{abstract}
Radiation-induced cranial nerve palsy (RICNP) is a severe long-term complication in patients with head and neck cancer following high-dose radiation therapy (RT). We present the case report of a patient with bilateral RICNP of the hypoglossal and vagus cranial nerves (XII/X) following postoperative RT in the era prior to the introduction of intensity-modulated RT (IMRT), and an analysis of our IMRT patient cohort at risk including the case of a XII RICNP. A total of 201 patients whose glosso-pharyngeal (IX), X and XII cranial nerves had been exposed to $>65$ Gy definitive IMRT in our institution between January, 2002 and December, 2012 with or without systemic therapy, were retrospectively identified. A total of 151 patients out of 201 fulfilling the following criteria were included in the analysis: Locoregionally controlled disease, with a follow-up (FU) of $>24$ months and $>65$ Gy exposure of the nerves of interest. So far, one of the assessed 151 IMRT patients at risk exhibited symptoms of RICNP after 6 years. The mean/median FU of the entire cohort was $71 / 68$ months (range, 27-145). The results were compared with literature reports. In conclusion, RICNP appears to be a rare complication. However, a longer FU and a larger sample size are required to draw reliable conclusions on the incidence of RICNP in the era of IMRT.
\end{abstract}

\section{Introduction}

Radiation-induced cranial nerve palsy (RICNP) is a severe long-term effect observed in patients treated with high-dose

Correspondence to: Professor Gabriela Studer, Department of Radiation Oncology, University Hospital Zurich, Rämistrasse 100, 8091 Zurich, Switzerland

E-mail: gabriela.studer@usz.ch

${ }^{*}$ Contributed equally

Key words: radiation-induced cranial nerve palsy, lower cranial nerves, radiation tolerance, intensity-modulated radiation therapy radiation therapy (RT) for nasopharyngeal carcinoma, with an incidence ranging between 3.7 and $30.9 \%$ in the literature (1-9).

Several studies have reported that the lower cranial nerves (CNs) are more susceptible to radiation damage (1-3). RICNP significantly compromises the quality of life: Damage of the glossopharyngeal nerve (IX) causes loss of sensation in the pharynx and decreased salivation; palsy of the vagus nerve (X) leads to impaired parasympathetic functions of almost all organs; and palsy of the hypoglossal nerve (XII) causes complete paralysis of the ipsilateral side of the tongue.

The aim of this study was to present the detailed clinical findings of two patients with RICNP following conventional three-dimensional (3D) RT and IMRT, respectively, and to analyze the IMRT cohort at risk (high radiation dose delivered to the upper level 2 and $7 \mathrm{~b}$ lymph node stations, where the nerves IX-XII pass through), in order to assess the incidence of RICNP in IMRT.

\section{Case reports}

Case report 1 (3D-RT). In June 1999, a 50-year-old male patient was referred to our department for postoperative RT for a squamous cell carcinoma of the right tonsil with unilateral lymph node metastases, staged as pT3 (4.2 cm), pN2b (1/29, Rouvière node), M0, R0 (minimal resection margin, $2 \mathrm{~mm}$ ), G3.

The patient had previously undergone trans-cervical oropharyngectomy with neck dissection of ipsilateral level 1-3 lymph nodes. Reconstruction of the defect was performed with a free radialis flap.

RT planning was performed using a computed tomography (CT)-based 3D conformal radiation technique. Adjuvant RT was performed according to the following concomitant boost (CCB) schedule: A first series with $20 x 1.8$ to a total of 36 Gy using laterally opposed photon beams, followed by spinal cord sparing electrons to the dorsal lymphatic pathways combined with anterior lateral opposed photon beam to a total of $50.4 \mathrm{~Gy}$. The CCB was performed with $9 \times 1.5$ Gy as a 2nd fraction/day, once/week. Unfortunately, the electron isodose distribution is no longer available, as the former treatment planning system has been replaced by other systems. In an attempt to reconstruct the 
A

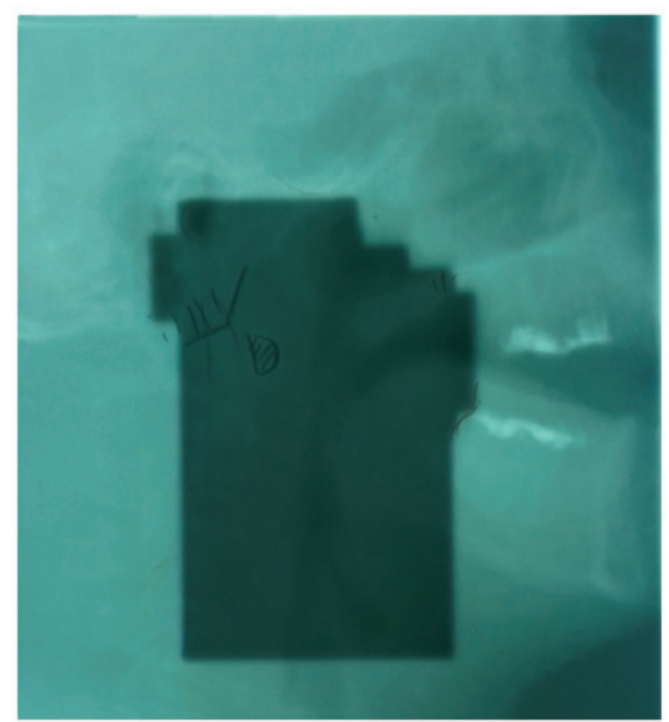

B

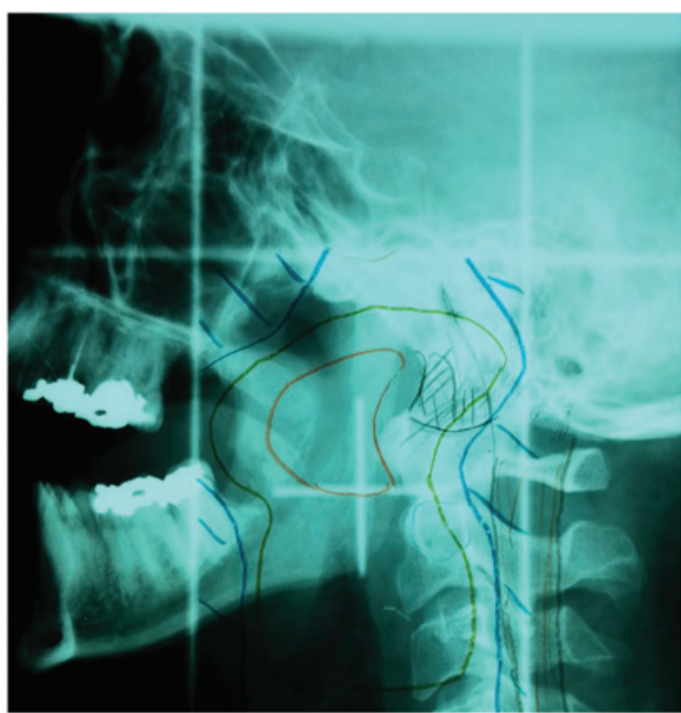

Figure 1. Case report. (A) Portal film of the boost (1999). Contoured: Anterior tubercle of the anterior atlas arc/atlanto-occipital joint. (B) Simulation film with primary gross tumor volumes drawn in 1999.

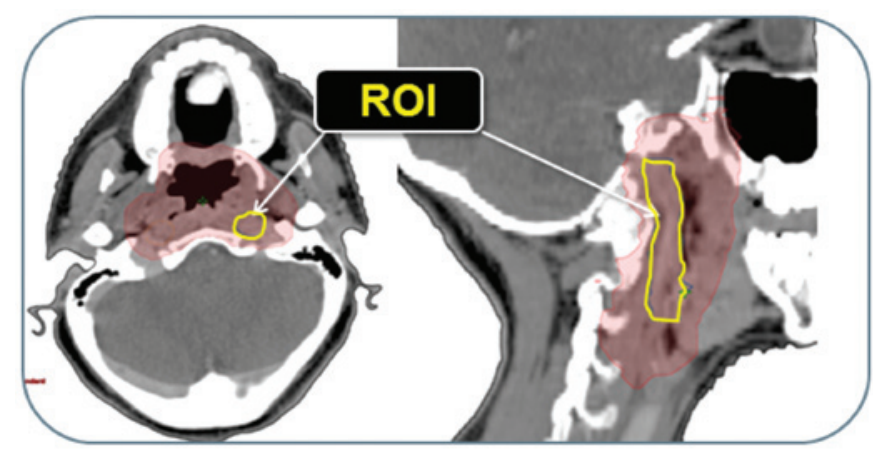

Figure 2. Contoured nerve region of interest (yellow outline) and planning target volume (red-shaded area). ROI, region of interest.

dose to the nerve region of interest (ROI) in this patient based on hard copy printouts from 1999, bilateral high-dose exposure was documented, as the primary lesion extended to both sides and there was significant nodal disease. The XII nerve was bilaterally exposed to the tumor dose, except for the most cranial aspect (2-3 cm from the hypoglossal canal), which was exposed to a lower dose ( $50-55 \mathrm{~Gy})$, which is usually well tolerated (Fig. 1). The patient underwent regular follow-up (FU) at our joint Otorhinolaryngology clinic (S.S., G.H.) until July, 2000. Late term tolerance to treatment has been good thus far, apart from a velopalatal insufficiency due to extensive palatal resection with consequent altered speech. Velopalatal reconstructive surgery was performed in July, 2000.

In May, 2011, the patient developed swallowing disturbances due to impaired tongue and laryngeal movement. Sufficient nutrition was ensured through a feeding tube. In June, 2011 the patient underwent transcervical cricomyotomy to reduce the resistance to swallowing, which achieved a minor improvement. At that time, bilateral XII nerve palsy was diagnosed. Centrally located causes, such as amyotrophic lateral sclerosis, were excluded. Following re-evaluation of the RT fields, XII nerve palsy was hypothesized to be a side effect of the bilateral irradiation 12 years prior.

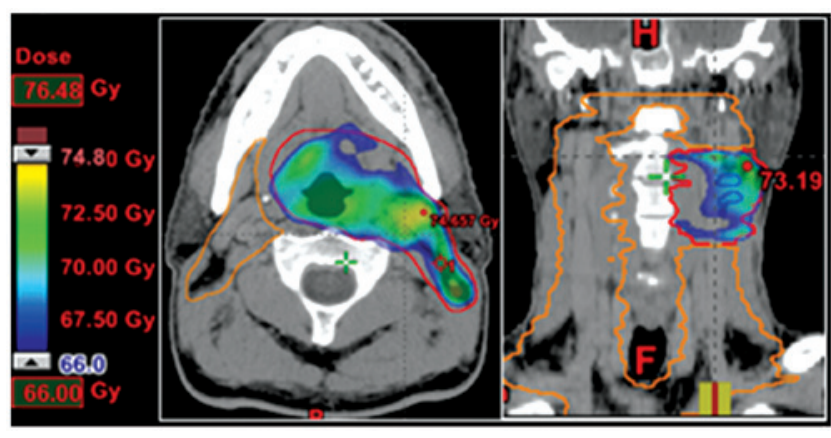

Figure 3. Case with left sided XII palsy 6 years post IMRT: depicted is the dose volume treated with $66-74 \mathrm{~Gy}$ in 30 fractions using simultaneously integrated boost (SIB).

Further extensive neurological and radiological investigations were performed to differentiate between RT-induced damage vs. other causes, which revealed an overall decreased motor neuron activity affecting other nerves, including the tibial and median nerves; to a certain extent, the progressive severe symptoms were considered to reflect the coexistence of radiation-related and independent neuronal disorders. However, in August, 2014, the patient was diagnosed with bilateral X nerve paresis with nocturnal stridor, feeding tube dependency and almost completely paralyzed tongue muscles, which supported the radiogenic etiology of the symptoms. To date, the patient remains tumor-free (September, 2014).

Care report 2 (IMRT). A 46-year-old patient underwent definitive SIB-IMRT with 30x2.2 Gy/fraction (66 Gy mean dose) for a left-sided cT1cN1 base of tongue carcinoma. Six years later he was diagnosed with an ipsilateral brachial plexopathy, which was due to a disc herniation C3/4 and C5/6, and a left XII nerve RICNP grade 2-3, for which other etiology with the exception of RT was excluded. The $2.2 \mathrm{~Gy} / \mathrm{f}$ SIB-IMRT schedule was used for several other patients in the initial IMRT implementation phase. The Dmax point dose in this patient was 74 Gy (Fig. 3). Two years later, the patient 
Table I. Patient and treatment parameters.

\begin{tabular}{|c|c|}
\hline Parameters & Values \\
\hline Patients, no. & 151 \\
\hline Cervical sides at risk, no. & 188 \\
\hline \multicolumn{2}{|l|}{ Primary tumor location, no. } \\
\hline Oropharynx & 75 \\
\hline Nasopharynx & 27 \\
\hline Hypopharynx & 30 \\
\hline Supraglottic area & 11 \\
\hline Oral cavity & 4 \\
\hline Glottic & 3 \\
\hline Unknown primary & 1 \\
\hline Gender ratio (female:male) & $1: 3.5$ \\
\hline Mean age, years (range) & $58(16-84)$ \\
\hline $\begin{array}{l}\text { Follow-up, months } \\
\text { mean/median (range) }\end{array}$ & $71 / 68(27-145)$ \\
\hline \multicolumn{2}{|l|}{ T stage, $\%$} \\
\hline 1 & 11 \\
\hline 2 & 24 \\
\hline 3 & 25 \\
\hline 4 & 39 \\
\hline Unknown primary & 1 \\
\hline \multicolumn{2}{|l|}{$\mathrm{N}$ stage, $\%$} \\
\hline 0 & 6 \\
\hline $1-2 b$ & 58 \\
\hline $2 c$ & 29 \\
\hline 3 & 7 \\
\hline Systemic therapy, no. & 82 \\
\hline
\end{tabular}

succumbed to an aggressive NHL, and therefore, no further RICNP FU is available.

\section{IMRT cohort}

In order to assess the incidence of RICNP in the IMRT era, we identified patients at risk treated in our department with normofractionated/slightly hypofractionated (2.0/2.11/2.2 Gy/f) IMRT between January, 2002 and December, 2012. All the patients were irradiated with 6-MV photons, with 66-70 Gy, 5 days/week, using simultaneous-integrated boost IMRT as previously described (10). The inclusion criteria were as follows: Patients with locoregionally controlled disease who received $>65$ Gy to the lower $\mathrm{CN}$ region with a $\mathrm{FU}$ of at least 24 months. These criteria were met by 151 patients treated with definitive IMRT, with or without additional chemotherapy. Focus was on the lower CNs, as they are the CNs most exposed to high radiation dose. The patient characteristics are summarized in Table I.

The corresponding anatomic ROI was retrospectively contoured in the planning CT by B.Y. and reviewed by C.G. (Fig. 2). The anatomic ROI was drawn from the base of the skull through the carotid sheath above the hyoid bone, where the CNs IX-XII are located between the internal jugular vein and

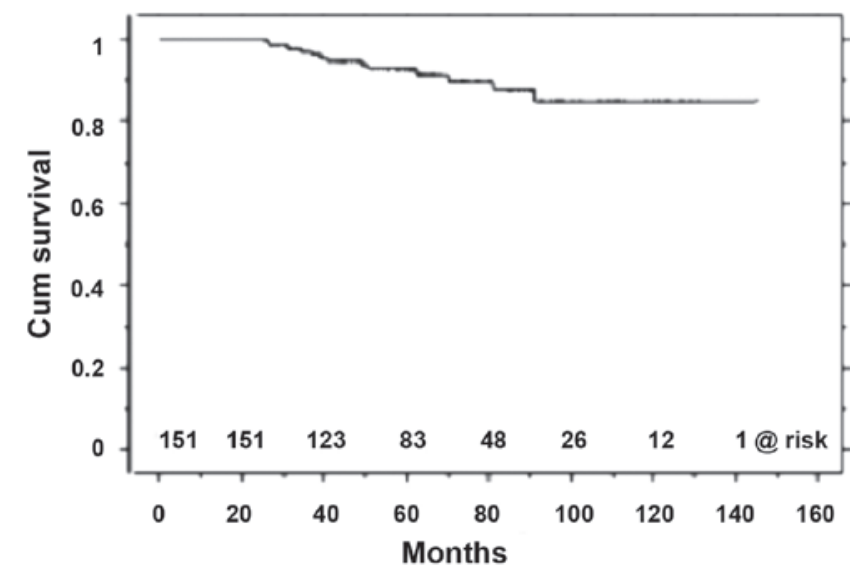

Figure 4. Overall survival curve of intensity-modulated radiation therapy cohort. Cum, cumulative.

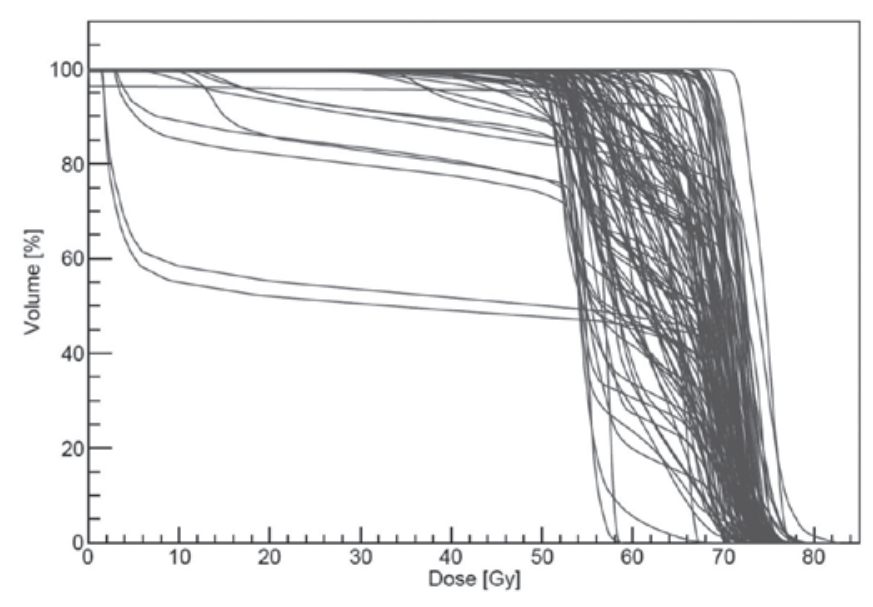

Figure 5. Dose-volume histogram of the nerve regions of interest (ROIs) in the assessed intensity-modulated radiation therapy cohort (see also Fig. 2) [absolute ROI volumes: Mean/median 9.4/9.0 cc (range, 5.5-13.9 cc)].

internal carotid artery down to the thyroid cartilage, which is approximately in the upper border of C4 (11). The space in the level of 2 nd and 3rd cervical vertebrae included the common carotid artery inferiorly and internal carotid artery superiorly, internal jugular vein and the IX, X, XI and XII cranial nerves. Due to the limited resolution of the planning CT, it was not possible to draw each of the lower CNs separately.

All the patients were followed up on a weekly basis during the RT period by a radiation oncologist. Regular FU visits were conducted in our joint clinic (Department of Otorhinolaryngology, Head and Neck Surgery, University Hospital of Zurich) and, in part, at a private clinic. The institutional standards for patient assessment included physical examination and flexible fiberoptic endoscopy every $\sim 2$ months during the first year of FU, every 3 months during the second and third years and every 6 months during the fourth and fifth years. In case of suspicious findings or clinical symptoms, further investigations were performed, including magnetic resonance imaging, positron emission tomography-CT, or fine-needle aspirations. Clinical examination to diagnose or exclude RICNP was routinely performed, based on clinical history and physical 


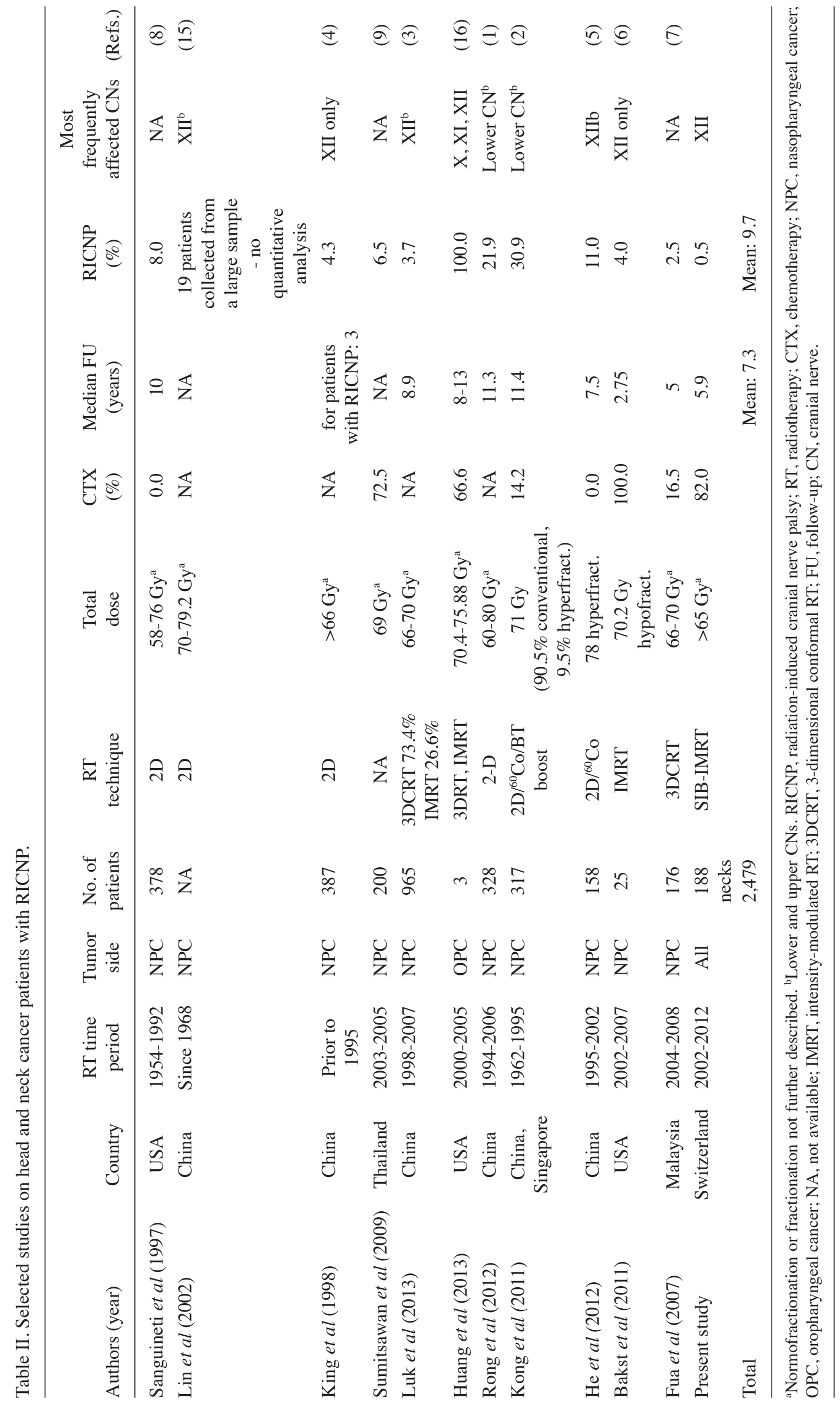


examination in our Otorhinolaryngology joint clinic. As CNP is a clinical disorder with distinct subjective symptoms, affected patients were reliably diagnosed during the regular FU visits; undetected cases were unlikely in the assessed cohort undergoing regular FU.

Systemic chemotherapy was administered in combination with IMRT when indicated [cisplatin weekly $40 \mathrm{mg} / \mathrm{m}^{2}$ (82\%) and/or cetuximab $400 \mathrm{mg} / \mathrm{m}^{2}$ loading dose followed by $250 \mathrm{mg} / \mathrm{m}^{2}$ weekly during RT, according to Bonner et al (12)]. To date, one of 151 patients in the IMRT cohort has developed lower CNP after 6 years post IMRT (see Case report 2). The overall survival of the IMRT cohort is depicted in Fig. 4. A total of 114 patients with unilateral and 37 patients with bilateral exposure [total: $114+(2 \times 37)=188$ ROIs in 151 individuals] were included in this analysis. The dose-volume histograms of all 188 ROIs are shown in Fig. 5. The absolute volumes of the ROIs were mean/median 9.4/9.3 cc (range, 5.5-14.5 cc). The mean dose was 65.45 Gy (range, 46.26-75.42 Gy) and the mean maximum dose was $75.42 \mathrm{~Gy}$ (range, 59.7-108.13 Gy). A total of 55,37 and $8 \%$ of the patients were followed up for 5,7 and 10 years, respectively. The mean/median duration of $\mathrm{FU}$ of the entire cohort was 71/68 months (range, 27-145 months).

\section{Discussion}

The presented case with bilateral CN XII palsy due to radiation exposure in the pre-IMRT era prompted us to analyze our IMRT cohort at risk. There was one (1) RICNP case among 151 assessed patients treated with definitive IMRT in our institution, after a mean FU of 71 months.

There were certain limitations to this analysis, mainly due to its retrospective approach and the relatively short observation time.

A crucial point when evaluating RICNP rates is the duration of observation of the assessed cohorts, as the risk for such events changes with longer FU. For RT-induced brachial plexopathy, the risk was found to be constant over a considerable portion of breast cancer patients' life (13). Johansson et al (14) retrospectively analyzed the latency for RT-induced neuropathy in breast cancer patients and found that the incidence at 5 years did not represent the full spectrum of injuries.

Over the last few years, certain study groups published results focusing on RICNP in patients with nasopharyngeal $(1-4,15)$ and oropharyngeal cancer (16). In those reports, the rate of RICNP ranges between 3.7 and 30.9\% (Table II). As summarized in Table II, the techniques and doses of RT and the frequency of chemotherapy application exhibited significant variations, which may be responsible for the wide range of RICNP incidence. The median FU time in the aforementioned studies ranged between 7.5 and 11.4 years. Rong et al (1) reported a mean latency for RICNP of 5.85 years. Kong et al (2) demonstrated that, among all patients who developed RICNP, $\sim 10 \%$ developed RICNP during every 5 years. The cumulative incidence for lower RICNP was 5.7, 17.4, 27.1 and $37.3 \%$ at 5, 10, 15 and 20 years, respectively. Another study group focusing on XII CNP reported the development of RICNP in 8 of the 17 affected patients within the first 50 months following RT completion (4).

Several risk factors for RICNP have been described: Kong et al (2) identified initial CNP at diagnosis, chemotherapy, total radiation dose and upper neck fibrosis as independent risk factors for developing RICNP. Luk et al (3) also found CNP at diagnosis to be a prognostic factor. In our cohort, $82 \%$ of the patients received systemic therapy and none of the included patients presented with CNP prior to treatment initiation. The majority of the studies on RICNP included patients prior to the availability of IMRT (Table II). IMRT is known to improve the therapeutic ratio and/or treatment tolerance in patients with head and neck cancer $(17,18)$ by facilitating sparing of normal tissue. In a prospective trial analyzing hypofractionated IMRT with chemotherapy for nasopharyngeal cancer, Bakst et al (6) only reported $4 \%$ RICNP.

In conclusion, our results and the general knowledge of the tissue sparing ability of IMRT indicate that modern RT techniques may affect the incidence of RICNP by exposing shorter parts of the nerves to high radiation doses and by causing less extensive muscle and soft tissue fibrosis close to the CNs, which was also considered to be partly responsible for the pathogenesis of RICNP. Applying IMRT, physicians must bear in mind that 'hot spots' in CN regions must be avoided (11). Hypofractctionation $>2.11 \mathrm{~Gy} / \mathrm{f}$ may result in a higher risk for RICNP due to hot spots. RT-induced nerve damage is known for a potentially long latency till clinical appearance (19).

Modulated tissue-sparing RT techniques may have the potential to reduce the incidence of RICNP. Longer FU is required to support this hypothesis.

\section{References}

1. Rong X, Tang Y, Chen M, Lu K and Peng Y: Radiation-induced cranial neuropathy in patients with nasopharyngeal carcinoma. A follow-up study. Strahlenther Onkol 188: 282-286, 2012.

2. Kong L, Lu JJ, Liss AL, Hu C, Guo X, Wu Y and Zhang Y: Radiation-induced cranial nerve palsy: A cross-sectional study of nasopharyngeal cancer patients after definitive radiotherapy. Int J Radiat Oncol Biol Phys 79: 1421-1427, 2011.

3. Luk YS, Shum JS, Sze HC, Chan LL, Ng WT and Lee AW: Predictive factors and radiological features of radiation-induced cranial nerve palsy in patients with nasopharyngeal carcinoma following radical radiotherapy. Oral Oncol 49: 49-54, 2013.

4. King AD, Leung SF, Teo P, Lam WW, Chan YL and Metreweli C: Hypoglossal nerve palsy in nasopharyngeal carcinoma. Head Neck 21: 614-619, 1999.

5. He X, Ye M, Guo X, Pan Z, Zhang Z, He S and Liu T: Treatment outcome of patients with stages I-II nasopharyngeal carcinoma after late course accelerated hyperfractionation radiotherapy alone. Oral Oncol 48: 1058-1063, 2012.

6. Bakst RL, Lee N, Pfister DG, Zelefsky MJ, Hunt MA, Kraus DH and Wolden SL: Hypofractionated dose-painting intensity modulated radiation therapy with chemotherapy for nasopharyngeal carcinoma: A prospective trial. Int J Radiat Oncol Biol Phys 80: 148-153, 2011.

7. Fua TF, Corry J, Milner AD, Cramb J, Walsham SF and Peters LJ: Intensity-modulated radiotherapy for nasopharyngeal carcinoma: Clinical correlation of dose to the pharyngo-esophageal axis and dysphagia. Int J Radiat Oncol Biol Phys 67: 976-981, 2007.

8. Sanguineti G, Geara FB, Garden AS, Tucker SL, Ang KK, Morrison WH and Peters LJ: Carcinoma of the nasopharynx treated by radiotherapy alone: Determinants of local and regional control. Int J Radiat Oncol Biol Phys 37: 985-996, 1997.

9. Sumitsawan Y, Chaiyasate S, Chitapanarux I, Anansuthiwara M, Roongrotwattanasiri K, Vaseenon V and Tooncam H: Late complications of radiotherapy for nasopharyngeal carcinoma. Auris Nasus Larynx 36: 205-209, 2009.

10. Peponi E, Glanzmann C, Kunz G, Renner C, Tomuschat K and Studer G: Simultaneous integrated boost intensity-modulated radiotherapy (SIB IMRT) in nasopharyngeal cancer. Strahlenther Onkol 186: 135-142, 2010. 
11. Mourad WF, Young BM, Young R, et al: Clinical validation and applications for CT-based atlas for contouring the lower cranial nerves for head and neck cancer radiation therapy. Oral Oncol 49: 956-963, 2013

12. Bonner JA, Harari PM, Giralt J, et al: Radiotherapy plus cetuximab for squamous-cell carcinoma of the head and neck. N Engl J Med 354: 567-578, 2006.

13. Bajrovic A, Rades D, Fehlauer F, Tribius S, Hoeller U, Rudat V, Jung $\mathrm{H}$ and Alberti W: Is there a life-long risk of brachial plexopathy after radiotherapy of supraclavicular lymph nodes in breast cancer patients? Radiother Oncol 71: 297-301, 2004.

14. Johansson S, Svensson H and Denekamp J: Dose response and latency for radiation-induced fibrosis, edema, and neuropathy in breast cancer patients. Int J Radiat Oncol Biol Phys 52: 1207-1219, 2002.

15. Lin YS, Jen YM and Lin JC: Radiation-related cranial nerve palsy in patients with nasopharyngeal carcinoma. Cancer 95: 404-409, 2002.
16. Huang AT, Song S, Dominguez LM, Nguyen J, Goldman RA and Reiter ER: Delayed lower cranial neuropathies following primary radiotherapy for oropharyngeal squamous cell carcinoma. Laryngoscope 123: 1207-1209, 2013.

17. May JT, Rao N, Sabater RD, et al: Intensity-modulated radiation therapy as primary treatment for oropharyngeal squamous cell carcinoma. Head Neck 35: 1796-1800, 2013.

18. Studer G, Linsenmeier C, Riesterer O, et al: Late term tolerance in head neck cancer patients irradiated in the IMRT era. Radiat Oncol 8: 259, 2013.

19. Studer G, Stocker DN, Loewenich KF and Glanzmann C: 40/42Gy in 13 fractions: a safe dose for the brachial plexus. J Nucl Med Radiat Ther 5: 168, 2014. 